\title{
Symbolic Pregression: Discovering Physical Laws from Distorted Video
}

\author{
Silviu-Marian Udrescu, Max Tegmark \\ Dept. of Physics \& Center for Brains, Minds $\&$ Machines, \\ Massachusetts Institute of Technology, Cambridge, MA 02139; sudrescu@mit.edu
}

(Dated: September 14, 2020)

\begin{abstract}
We present a method for unsupervised learning of equations of motion for objects in raw and optionally distorted unlabeled synthetic video. We first train an autoencoder that maps each video frame into a low-dimensional latent space where the laws of motion are as simple as possible, by minimizing a combination of non-linearity, acceleration and prediction error. Differential equations describing the motion are then discovered using Pareto-optimal symbolic regression. We find that our pre-regression ("pregression") step is able to rediscover Cartesian coordinates of unlabeled moving objects even when the video is distorted by a generalized lens. Using intuition from multidimensional knot-theory, we find that the pregression step is facilitated by first adding extra latent space dimensions to avoid topological problems during training and then removing these extra dimensions via principal component analysis. An inertial frame is auto-discovered by minimizing the combined equation complexity for multiple experiments.
\end{abstract}

\section{INTRODUCTION}

A central goal of physics and science more broadly is to discover mathematical patterns in data. For example, after four years of analyzing data tables on planetary orbits, Johannes Kepler started a scientific revolution in 1605 by discovering that Mars' orbit was an ellipse [1. There has been great recent progress in automating such tasks with symbolic regression: discovery of a symbolic expression that accurately matches a given data set 2 23. Open-source software now exists that can discover quite complex physics equations by combining neural networks with techniques inspired by physics and information theory [22, 23].

However, there is an important underlying problem that symbolic regression does not solve: how to decide which parameters of the observed data we should try to describe with equations. Eugene Wigner famously stated that "the world is very complicated and ... the complications are called initial conditions, the domains of regularity, laws of nature" [24, so how can the discovery of these regularities be automated? In Figure 1, how can an unsupervised algorithm learn that to predict the next video frame, it should focus on the $x$ - and $y$-coordinates of the rocket, not on its color or on the objects in the background? More generally, given an evolving data vector with $N$ degrees of freedom, how can we auto-discover which $n<N$ degrees of freedom are most useful for prediction? Renormalization addresses this question in a particular context, but we are interested in generalizing this. In Kepler's case, for example, the raw data corresponds to two-dimensional sky images: how can a computer algorithm presented with a series of images with say $N=10^{6}$ pixels automatically learn that the most useful degrees of freedom are the $n=2$ position coordinates of the small reddish dot corresponding to Mars?

The goal of our paper is to tackle this pre-regression problem, which we will refer to this as "pregression" for brevity. Automated pregression enables laws of motion to be discovered starting with raw observational data such as videos. This can be viewed as a step toward unsupervised learning of physics, whereby an algorithm learns from raw observational data without any human supervision or prior knowledge [25]27].
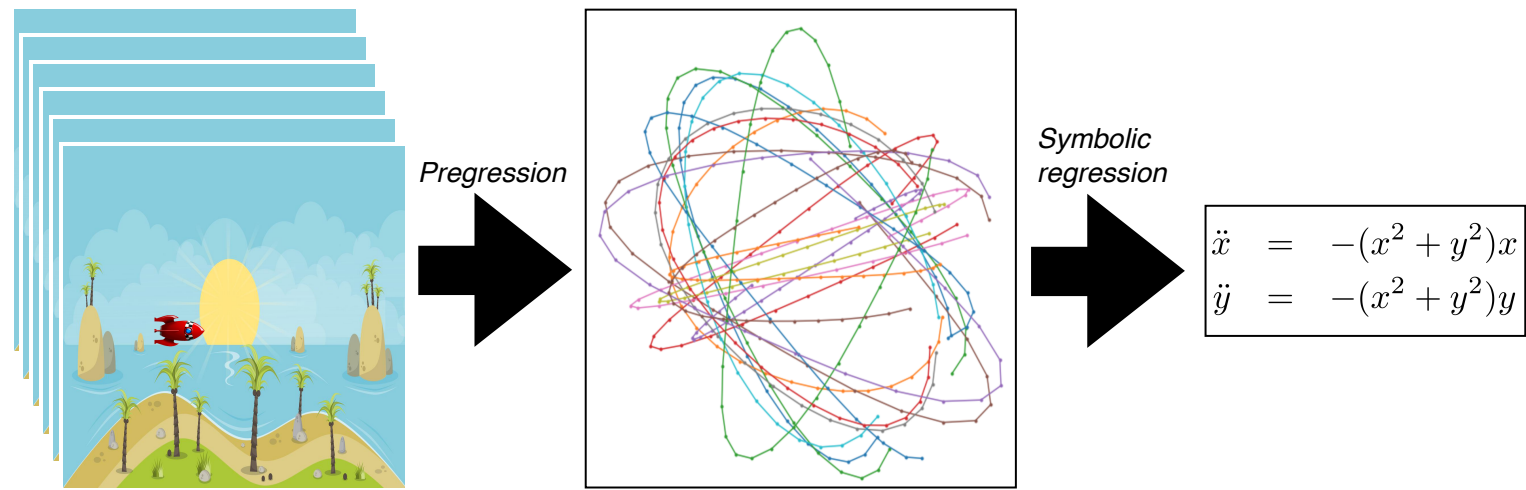

FIG. 1: Our pregression algorithm seeks to autoencode a sequence of video frames (left) corresponding to a specific type of motion into a low-dimensional latent space (middle) where the laws of motion (right) are as simple as possible, in this example those of a quartic oscillator. In the middle figure each point corresponds to the $\mathrm{x}$ and $\mathrm{y}$ of the rocket in a given frame, while points having the same color and connected by a line belong to the same trajectory. 
There has been impressive recent progress on using neural networks for video prediction 28, 42 and more general physics problems 27, 43 48]. However, these machine-learned models tend to be inscrutable black boxes that provide their human users with limited understanding. In contrast, the machine learning approach in this paper aspires to intelligible intelligence, i.e., learning a model of the system that is simple enough for a human user to understand. Such intelligibility (pursued in, e.g., [26, 27] 49, 52]) is a central goal of physics research, and has two advantages:

1. Understanding how a model works enables us to trust it more, which is particularly valuable when AI systems make decisions affecting peoples lives [53 56].

2. Simple intelligible models such as the laws of physics tend to yield more accurate and generalizable predictions than black-box over-parametrized fits, especially over long timescales. This is why spacecraft navigation systems use Newton's law of gravitation rather than a neural-networkbased approximation thereof.

The video prediction papers most closely related to the present work take one of two approaches. Some improve accuracy and intelligibility by hardcoding coordinate-finding or physics elements by hand to help learn e.g. rigid-body motion [57, physical object properties or partial differential equations [58, 59]. The alternative tabula rasa approach assumes no physics whatsoever and attempts to learn physical object properties [60, object positions 61, 62, object relations [63] and time evolution 64,66 by learning a lowdimensional representation or latent space which is unfortunately too complex or inscrutable to allow discovery of exact equations of motion. The present paper builds on this tabula rasa approach; our key contribution is to automatically simplify the latent space, using ideas inspired by general relativity and knot theory, to make the dynamics simple enough for symbolic regression to discover equations of motions.

The rest of this paper is organized as follows. In Section II] we present our algorithm. In Section III we test it on simulated videos (such as the flying rocket example in Figure 1 for motion in a force-free environment, a gravitational field, a magnetic field, a harmonic potential and a quartic potential. We also test the effects of adding noise and geometric image distortion. We summarize our conclusions and discuss future challenges in Section IV

\section{METHOD}

The goal of our method is to start with raw video sequences of an object moving in front of some static background, and to, in a fully unsupervised manner (with no input besides the raw video) discover the differential equation governing the object's motion. Our algorithm consists of two parts:

1. a neural-network-based pregression step that learns to map images into a low-dimensional latent space representing the physically relevant parameters (degrees of freedom), and

2. a symbolic regression step that discovers the law of motion, i.e., the differential equation governing the time-evolution of these parameters.

\section{A. Learning the latent space}

Abstractly, we can consider each video frame as a single point in an $N$-dimensional space, where $N$ is the number of color channels ( 3 in our case) times the number of pixels in each image. If the

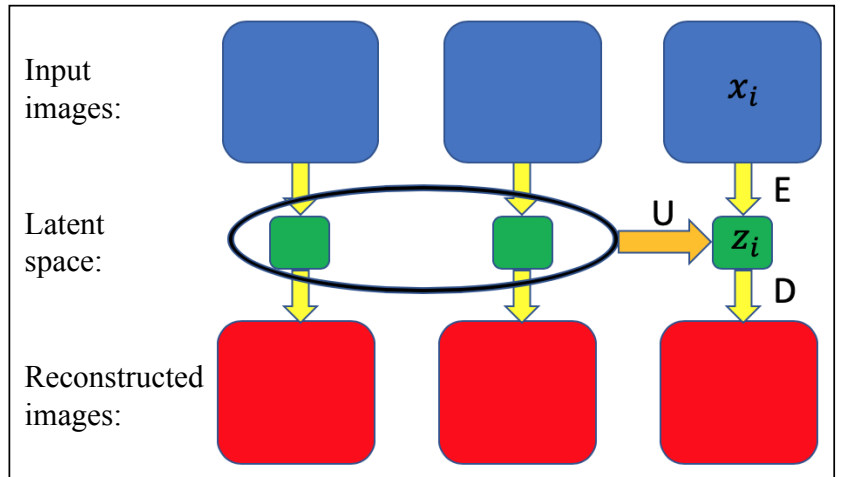

FIG. 2: Architecture of our neural network: an encoder $E$ that maps images $\mathbf{x}_{i}$ into latent space vectors $\mathbf{z}_{i}$, a decoder $D$ that maps latent space vectors $\mathbf{z}_{i}$ back into images $\mathbf{x}_{i}$, and an evolution operator $U$ that predicts the next latent space vector from the two previous ones.

motion involves only $n \ll N$ degrees of freedom (for example, $n=$ 2 for a rigid object moving without rotating in two dimensions), then all observed points in the $N$-dimensional space lie on some $n$ dimensional submanifold that we wish to discover, parametrized by an $n$-dimensional parameter vector that we can consider as a point in an $n$-dimensional latent space. Our neural network architecture for learning the latent space is shown in Figure 1 and consists of three separate feedforward neural networks:

1. An encoder $E$ that maps images $\mathbf{x}_{i} \in \mathbb{R}^{N}$ into latent space vectors $\mathbf{z}_{i} \in \mathbb{R}^{n}$,

2. a decoder $D$ that maps latent space vectors $\mathbf{z}_{i}$ into images $\mathbf{x}_{i}$, and

3. an evolution operator $U$ that predicts the next latent space vector $\mathbf{z}_{i}$ from the two previous ones (two are needed to infer velocities). ${ }^{1}$

The encoder-decoder pair forms an autoencoder [67- 75 that tries to discover the $n$ most dynamically relevant parameters from each movie frame, from which it can be reconstructed as accurately as possible.

\section{B. Quantifying simplicity}

It is tempting to view the results of our pregression algorithm as rather trivial, merely learning to extract $x-$ and $y$ - coordinates of objects. This would be incorrect, however, since we will see that the pregression discovers simple physical laws even from video images that are severely warped, as illustrated in Figure 3 where the learned latent space is a complicated non-linear function of the Cartesian coordinates. The basic reason for this is that Figure 1 makes no mention of any preferred latent-space coordinate system. This reparametrization invariance (a core feature of general relativity) is a double-edged sword, however: a core challenge that we must overcome is that even if the system can be described by a

1 The two last images are needed because the laws of physics are second order differential equations that can be transformed into second order difference equations; our method trivially generalizes to using the last $T$ inputs for any choice $T=1,2,3, \ldots$ 
simple time-evolution $U$, the basic architecture in Figure 1 may discover something much more complicated. To see this, suppose that there is an autoencoder $(E, D)$ and evolution operator $U$ providing perfect image reconstruction and prediction, i.e., satisfying

$$
\begin{aligned}
& D\left(E\left(\mathbf{x}_{i}\right)\right)=\mathbf{x}_{i}, \\
& U\left(\mathbf{z}_{i-2}, \mathbf{z}_{i-1}\right)=\mathbf{z}_{i}
\end{aligned}
$$

and that $U$ is a fairly simple function. If we now deform the latent space by replacing $\mathbf{z}$ by $\mathbf{z}^{\prime} \equiv f(\mathbf{z})$ for some invertible but horribly complicated function $f$, then it is easy to see that the new mappings defined by

$$
\begin{aligned}
E^{\prime}(\mathbf{x}) & \equiv f(E(\mathbf{x})), \\
D^{\prime}\left(\mathbf{z}^{\prime}\right) & \equiv D\left(f^{-1}\left(\mathbf{z}^{\prime}\right)\right), \\
U^{\prime}\left(\mathbf{z}^{\prime}\right) & \equiv f\left(U\left(f^{-1}\left(\mathbf{z}^{\prime}\right)\right)\right)
\end{aligned}
$$

will still provide perfect autoencoding and evolution

$$
\begin{aligned}
& D^{\prime}\left(E^{\prime}\left(\mathbf{x}_{i}\right)\right)=\mathbf{x}_{i}, \\
& \left.U^{\prime}\left(\mathbf{z}_{i-2}, \mathbf{z}_{i-1}\right)\right)=\mathbf{z}_{i}
\end{aligned}
$$

even though the new evolution operator $U^{\prime}$ is now very complicated.
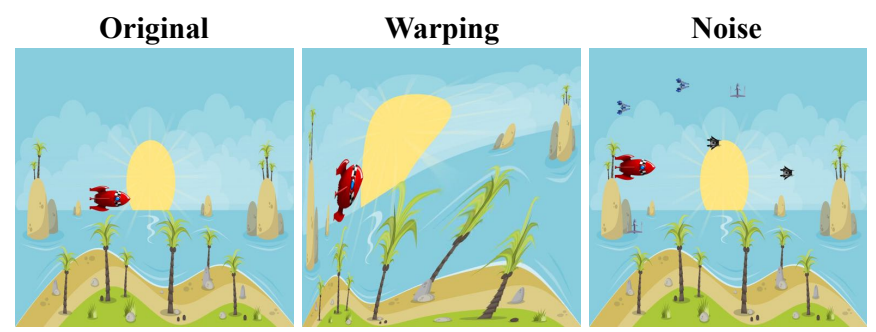

FIG. 3: Our method can discover simple laws of motion even if the true images (left) are severely warped (middle) or corrupted by superimposed noise in the form of smaller distractor rockets (right).

Not only can our architecture discover unnecessarily complicated solutions, but it by default will. We jocularly termed this the Alexander principle in honor of a child of one of the authors whose sense of humor dictated that he comply with requests in the most complicated way consistent with the instructions. We will face multiple challenges of this type throughout this paper, where our neural networks appeared humorously spiteful simply because they statistically find the most generic solution in a vast class of equally accurate ones.

To tackle this problem, we wish to add a regularization term to the loss function that somehow rewards simplicity and penalizes complexity, ideally in a way that involves as few assumptions as possible about the type of dynamics occurring in the video. Defining the $2 \mathrm{n}$-dimensional vector

$$
\mathbf{w}_{i} \equiv\left(\begin{array}{c}
\mathbf{z}_{i-2} \\
\mathbf{z}_{i-1}
\end{array}\right) \in \mathbb{R}^{2 n}
$$

we can view the evolution function $U(\mathbf{w})$ as a mapping from $\mathbb{R}^{2 n}$ to $\mathbb{R}^{n}$ that we wish to be as simple as possible. A natural physicsinspired complexity measure for $U$ is its curvature

$$
\mathcal{L}^{\text {curv }} \equiv R_{\mu \nu \beta}^{\alpha} R_{\alpha}^{\mu \nu \beta},
$$

defined as the squared Riemann tensor that is ubiquitous in differential geometry and general relativity, defined as

$$
\begin{aligned}
R_{\mu \nu \beta}^{\alpha} & \equiv \Gamma_{\nu \beta, \mu}^{\alpha}-\Gamma_{\mu \beta, \nu}^{\alpha}+\Gamma_{\mu \beta}^{\gamma} \Gamma_{\nu \gamma}^{\alpha}-\Gamma_{\nu \beta}^{\gamma} \Gamma_{\mu \gamma}^{\alpha} \\
\Gamma_{\mu \nu}^{\alpha} & \equiv \frac{1}{2} g^{\alpha \sigma}\left(g_{\sigma \mu, \nu}+g_{\sigma \nu, \mu}-g_{\mu \nu, \sigma}\right) \\
\mathbf{g} & \equiv \mathbf{J J}^{t}
\end{aligned}
$$

where $\mathbf{J}$ is the Jacobian of $U$, the matrix $\mathbf{g}$ is the induced metric on the latent space $\mathbb{R}^{n}$, indices are raised by multiplying by $\mathbf{g}^{-1}$, commas denote derivatives as in standard tensor notation, and the Einstein summation convention is used. Natural alternatives are the squared Ricci curvature $R^{\mu \nu} R_{\mu \nu}$ or the scalar curvature $R \equiv$ $g^{\mu \nu} R_{\mu \nu}$, where $R_{\mu \nu} \equiv R_{\mu \alpha \nu}^{\alpha}$.

Unfortunately, these curvature measures are numerically cumbersome, since they require taking 3rd derivatives of the neuralnetwork-defined function $U$ and the Riemann tensor has $n^{4}$ components. Fortunately, we find that a simpler measure of complexity performs quite well in practice, as reflected by the following loss function:

$$
\mathcal{L} \equiv \mathcal{L}^{\text {recon }}+\alpha \mathcal{L}^{\text {pred }}+\beta \mathcal{L}^{\text {nl }}+\gamma \mathcal{L}^{\text {acc }}
$$

These four terms are averages over all time steps $i$ of the following dimensionless functions:

$$
\begin{aligned}
\mathcal{L}_{i}^{\text {recon }} & \equiv \frac{\left|\mathbf{x}_{i}-D\left(E\left(\mathbf{x}_{i}\right)\right)\right|}{\left|\mathbf{x}_{i}\right|}, \\
\mathcal{L}_{i}^{\text {pred }} & \equiv \frac{\left.\mid \mathbf{z}_{i}-U\left(\mathbf{z}_{i-2}, \mathbf{z}_{i-1}\right)\right) \mid}{\left|\mathbf{z}_{i-1}-\mathbf{z}_{i-2}\right|}, \\
\mathcal{L}_{i}^{\text {nl }} & \left.\equiv \frac{1}{4 n^{3}}\left|\mathbf{z}_{i-1}-\mathbf{z}_{i-2}\right| \| \nabla \mathbf{J}\left(\mathbf{w}_{i}\right)\right) \|_{1}, \\
\mathcal{L}_{i}^{\text {acc }} & \equiv \frac{1}{n}|| U\left(\mathbf{w}_{i}\right)-\mathbf{M} \mathbf{w}_{i} \|_{1},
\end{aligned}
$$

$\alpha, \beta, \gamma$ are tunable hyperparameters, and $n$ is the dimensionality of the latent space. Here $\mathcal{L}^{\text {recon }}$ is the reconstruction error, $\mathcal{L}^{\text {pred }}$ is the prediction error, and both $\mathcal{L}^{\text {nl }}$ and $\mathcal{L}^{\text {acc }}$ are measures of the complexity of $U . \mathcal{L}^{\mathrm{nl}}$ is a measure of the nonlinearity of the mapping $U$, since its Jacobian $\mathbf{J}$ will be constant if the mapping is linear. Note that $\mathcal{L}^{\mathrm{nl}}=0$ implies that $\mathcal{L}^{\text {curv }}=0$, since if $\mathbf{J}$ is constant, then $\Gamma_{\mu \nu}^{\alpha}=0$ and the curvature vanishes. Physically, $\mathcal{L}^{\mathrm{nl}}=0$ implies that the dynamics is described by coupled linear difference equations, which can be modeled by coupled linear differential equations and encompass behavior such as helical motion in magnetic fields, sinusoidal motion in harmonic oscillator potentials and parabolic motion under gravity. $\mathcal{L}^{\text {acc }}$ is a measure of the predicted acceleration, since there is no acceleration if the mapping is $U(\mathbf{w})=\mathbf{M w}$, where

$$
\mathbf{M} \equiv(-\mathbf{I} 2 \mathbf{I}),
$$

and $\mathbf{I}$ is the $n \times n$ identity matrix. For example, $x_{i}=2 x_{i-1}-x_{i-2}$ gives uniform $1 \mathrm{D}$ motion (with $i$ indicating the time step at which the $x$ coordinate is recorded). An alternative implementation not requiring Jacobian gradient evaluation would be $\mathcal{L}_{i}^{\text {nl }} \equiv$ $\frac{1}{2 n^{2}}\left\|\mathbf{J}\left(\mathbf{w}_{i+1}\right)-\mathbf{J}\left(\mathbf{w}_{i}\right)\right\|_{2}^{2}$, and an alternative acceleration penalty would be $\left.\left.\mathcal{L}_{i}^{\text {acc }} \equiv \frac{1}{n} \mid U(\mathbf{0})\right)\left.\right|^{2}+\frac{1}{2 n^{2}} \| \mathbf{M}-\mathbf{J}\left(\mathbf{w}_{i}\right)\right) \|_{2}^{2}$.

\section{RESULTS}

\section{A. Latent space learning}

We first tested our algorithm for four physical systems obeying linear differential equations, corresponding to motion with no forces, in a gravitational field, in a magnetic field and in a $2 \mathrm{D}$ harmonic oscillator potential (see Figure 4). For each type of motion, we generated between 100 and 150 trajectories, with around 30 video frames each, corresponding to equally spaced, consecutive time steps. For each trajectory video, the shape of the rocket and the background were kept fixed, but the position of the rocket was changed according to the corresponding physical law of motion, starting with a random initial velocity and a random initial position within the image boundaries. Our training set thus contains a total of 30005000 images for each type of motion; sample trajectories are shown in Figure 4 (top), where each dot represents the $x$ - and $y$-coordinate 


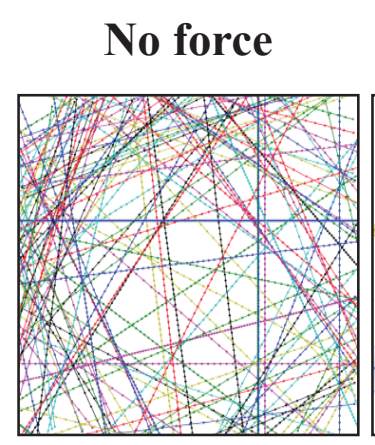

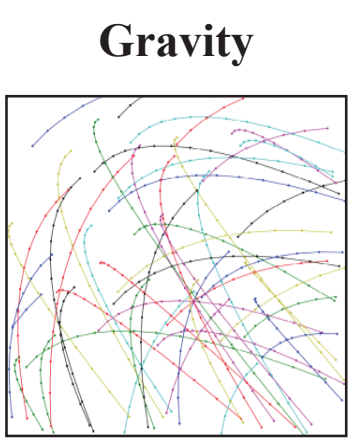
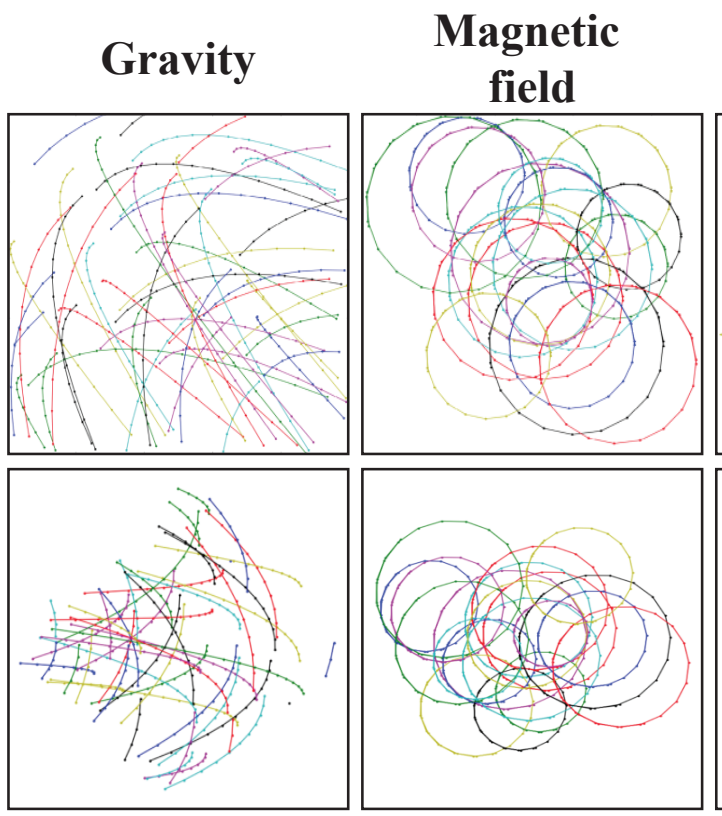

\section{D harmonic oscillator}
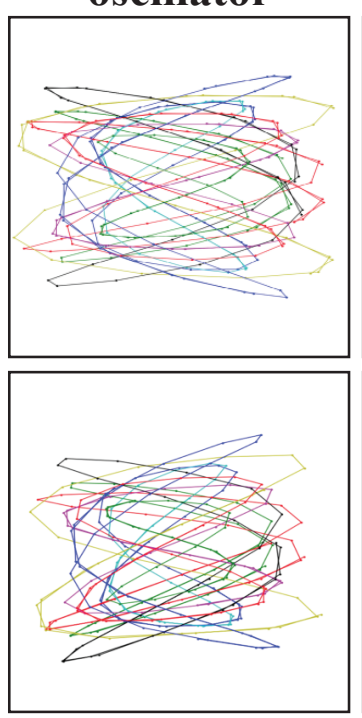

Quartic oscillator
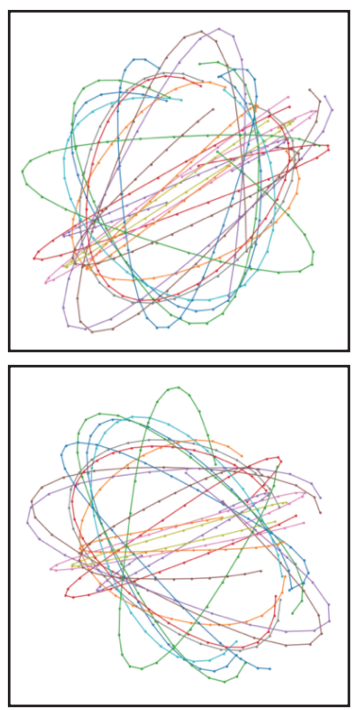

FIG. 4: Example of original (top) and discovered (bottom) trajectories in the latent spaces. In the top panel, each point represents the $x$ - and $y$-coordinates of the rocket in each frame. In the bottom panel, each point corresponds to the two main principal components discovered in the $5 \mathrm{D}$ latent space. In both cases, points of the same color and connected by a line belong to the same trajectory.

of the rocket in a given frame and points of the same color connected by a line belong to the same trajectory. After simulating the trajectories and generating a $1000 \times 1000$ pixel image of each video frame (Figure 1 for an example), we downsampled the image resolution to $64 \times 64$ pixels before passing them to our neural network.

The encoder network consists of five convolutional ReLU layers with kernel-size 4 and padding 1 , four with stride 2 followed by one with stride 1 . At the end there is a fully connected linear layer that reduces the output of the encoder to a vector of size equal to the dimension of the latent space. The number of channels goes from 3 for the input image to $32,32,64,64$ and 256 for the convolutional layers. The decoder network is a mirror image of the encoder in terms of layer dimensions, with the convolution layers replaced by deconvolution layers. The evolution operator has three fully connected 32-neuron hidden layers with softplus activation function and a linear $n$-neuron output layer. We implemented these networks using PyTorch using a batch size of 256 and the Adam optimizer. For these four linear types of motion, we set $\gamma=0$ and $\alpha=\beta=10^{-3}$ and trained for 4,000 epochs with a learning rate of $10^{-3}$, multiplying $\alpha$ and $\beta$ by 10 after every 1000 epochs. We then trained for 3,000 additional epochs while dividing the learning rate by 10 every 1,000 epochs.

Although our algorithm successfully learned useful 2D latent spaces (Figure 4 , bottom) and predicted images with $2 \%$ r.m.s. relative error that were visually nearly indistinguishable from the truth, this required overcoming two separate obstacles. We initially lacked the factor $\left|\mathbf{z}_{i-1}-\mathbf{z}_{i-2}\right|$ in equation [11, so by the Alexander principle, the neural network learned to drive the prediction loss $\mathcal{L}^{\text {pred }}$ toward zero by collapsing the latent space to minuscule size. The $\left|\mathbf{z}_{i-1}-\mathbf{z}_{i-2}\right|$-factor solves this problem by making the prediction loss invariant under latent space rescaling.

\section{B. Knot theory to the rescue}

The second obstacle is topological. If you drop a crumpled-up towel (a 2D surface in 3D space) on the floor, it will not land perfectly flat, but with various folds. Analogously, the space of all possible rocket images forms a highly curved surface in the $N$-dimensional space of images, so when a randomly initialized neural network first learns to map it into a 2D latent space, there will be numerous folds. For example, the left panel of Figure 5 shows 16 trajectories (each shown in a different color) corresponding to the rocket moving uniformly in straight lines. The middle panel shows these same trajectories (with the same colors as in the left panel) in the latent space first discovered by our neural network when we allowed only two latent space dimensions. Some pairs of trajectories which are supposed to be straight parallel lines (left panel) are seen to cross in a cat-like pattern in the latent space (middle panel) even though they should not cross. During training, the network tries to reduce prediction and complexity loss by gradually distorting this learned latent space to give trajectories the simplest possible shapes (straight lines in this case), but gets stuck and fails to unfold the latent space. This is because the reconstruction loss $\mathcal{L}^{\text {recon }}$ effectively causes distinct images to repel each other in the latent space: if two quite different rocket images get mapped to essentially the same latent-space point, then the decoder will epically fail for at least one. Unfolding would require temporarily moving one trajectory across another, thus greatly increasing the loss. This is analogous to topological defects in physics that cannot be removed because of an insurmountable energy barrier.

Fortunately, knot theory comes to the rescue: a famous theorem states that there are no $d$-dimensional knots in an $n$-dimensional space if $n>\frac{3}{2}(1+d)$ [76]. For example, you cannot tie your shoelaces $(d=1)$ if you live in $n=4$ dimensions. Our topological pregression problem corresponds to the inability of the neural network to untie a $d$-dimensional knot in $n$ dimensions, where $d$ is the dimensionality of the image submanifold of $\mathbb{R}^{N}(d=2$ for our examples). We therefore implemented the following solution, 

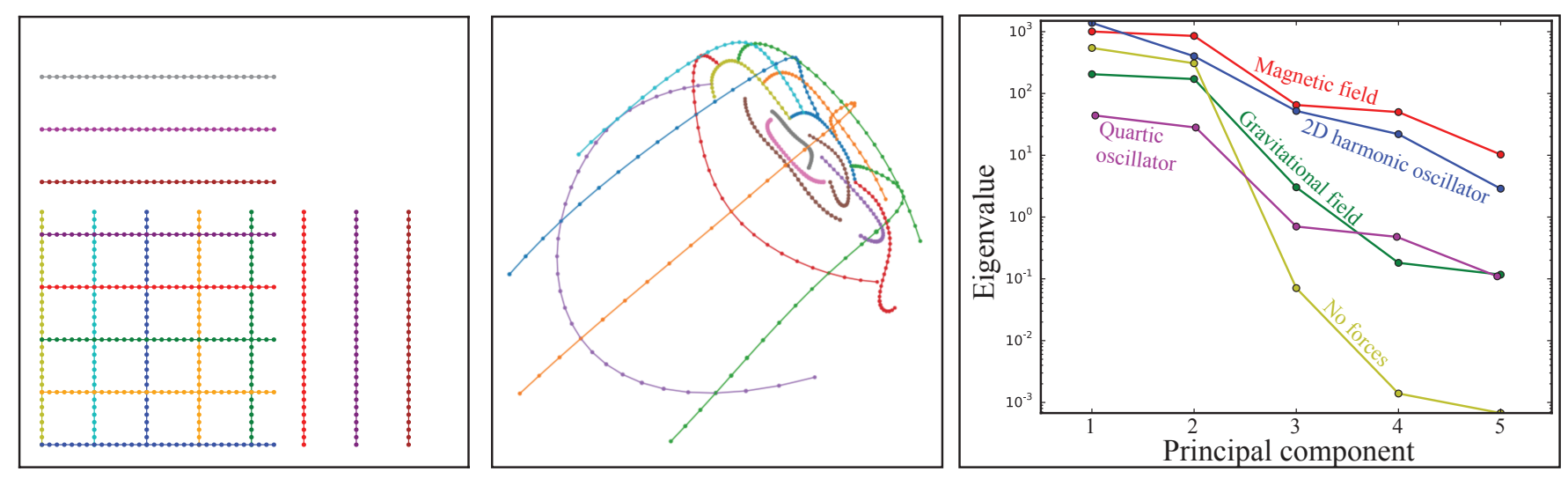

FIG. 5: The topological problems (middle) that prevented directly learning a 2-dimensional latent space (left) can be understood via knot theory and eliminated by instead discovering the two main principal components (right) in a learned 5-dimensional latent space. The left panel shows 16 force-free rocket trajectories, with points denoting the rocket center in each frame and points of the same color corresponds to the same trajectory. The middle panel shows the corresponding points $\mathbf{z}_{i}$ produced by our encoder network trained with a $2 \mathrm{D}$ latent space. The right panel shows the eigenvalues obtained from a PCA on a five-dimensional learned latent space, revealing that the latent space is rather 2-dimensional because two principal components account for most of the variance.

which worked well for all our examples: First run the pregression algorithm with a latent space of dimension $n^{\prime}>\frac{3}{2}(1+d)$ (we found $n^{\prime}=5$ to be enough for us) and then extract an $n$-dimensional latent space using principal component analysis. This corresponds to incentivizing the aforementioned towel to flatten out while still in the air and then rotating it to be parallel to the floor before landing. Figure 5 (right) shows that upon applying PCA to the points in the 5-dimensional latent space, two principal components dominate the rest (accounting for more than 90\% of the variance), revealing that all rocket images get mapped roughly into a $2 \mathrm{D}$ plane (Figure 4 in a 5D latent space.

\section{Nonlinear dynamics and the accuracy-simplicity tradeoff}

Increasing the two parameters $\beta$ and $\gamma$ in equation 9 penalizes complexity $\left(\mathcal{L}^{\mathrm{nl}}\right.$ and $\left.\mathcal{L}^{\text {acc }}\right)$ more relative to inaccuracy $\left(\mathcal{L}^{\text {recon }}\right.$ and $\left.\mathcal{L}^{\text {pred }}\right)$. For our quartic oscillator example (Figure 1 , achieving $\mathcal{L}^{\mathrm{nl}}=0$ is impossible and undesirable, since the correct dynamics is nonlinear with $\nabla \mathbf{J} \neq 0$, so we wish to find the optimal tradeoff between simplicity and accuracy. We did this by training as above for 7,000 epochs but setting $\beta=0$, then keeping $\gamma=\beta$ and further training 14 networks in parallel for a geometric series of $\beta$-values from 0.01 to 200 . These 14 networks were trained for 3,000 epochs with learning rate starting at $10^{-3}$ and dropping tenfold every 1,000 epochs.

Since, as mentioned above, there is a broad class of equally accurate solutions related by a latent space reparametrization $\mathbf{z} \mapsto f(\mathbf{z})$, we expect that increasing $\beta$ from zero to small values should discover the simplest solution in this class without decreasing prediction or reconstruction accuracy. This is the solution we want, in the spirit of Einstein's famous dictum "everything should be made as simple as possible, but not simpler". Further increasing $\beta$ should simplify the solution even more, but now at the cost of leaving this equivalence class, reducing accuracy. Our numerical experiment confirmed this expectation: we could increase regularization to $\beta=50$ (the choice shown in Figure 1) without significant accuracy loss, after which the inacuraccy started rising abruptly. It should be noted that a similar Pareto approach could be used for the other four linear types of motions, but in those cases, the Pareto frontier would be trivial, given that the right solution (minimum loss) corresponds to having no non-linearity (minimum complexity).

\section{Image warping and noise}

As mentioned in Section IIB the fact that our algorithm rewards simplicity in the evolution operator $U$ rather than the encoder/decoder pair should enable it to discover the simplest possible latent space even if the space of image $(x, y)$-coordinates is severely distorted. To test this, we replaced each image with color $c[x, y]$ (defined over the unit square) by a warped image $c^{\prime}[x, y]$ defined by

$$
\begin{aligned}
c^{\prime}[x, y] & \equiv c[g(x)+x(1-x) y, g(y)+y(1-y) x], \\
g(u) & \equiv u\left(11-18 u+12 u^{2}\right) / 5
\end{aligned}
$$

as illustrated in Figure 3 (middle panel), and analyzed the 3,000 warped video frames of the rocket moving in a magnetic field. As expected, the pregression algorithm recovered a non-warped latent space just as in Figure 4 so this extra complexity was entirely absorbed by the decoder/encoder, which successfully learned the warping function $c \mapsto c^{\prime}$ of equation 15 and its inverse.

We also tested the robustness of our pregression algorithm to noise in the form of smaller rockets added randomly to each video frame. We used 3 different types of distractor rockets as noise, and added between zero and 10 to each image as illustrated in Figure 3 (right panel). The result was that the pregression algorithm learned to reconstruct the latent space just as before, focusing only on the large rocket, and reconstructing images with the distractor rockets removed.

\section{E. Automatically discovering equations and inertial frames}

Let us now turn to the task of discovering physical laws that are both accurate and simple. Although the five rocket-motion examples took place in the same image space, the Alexander Principle 


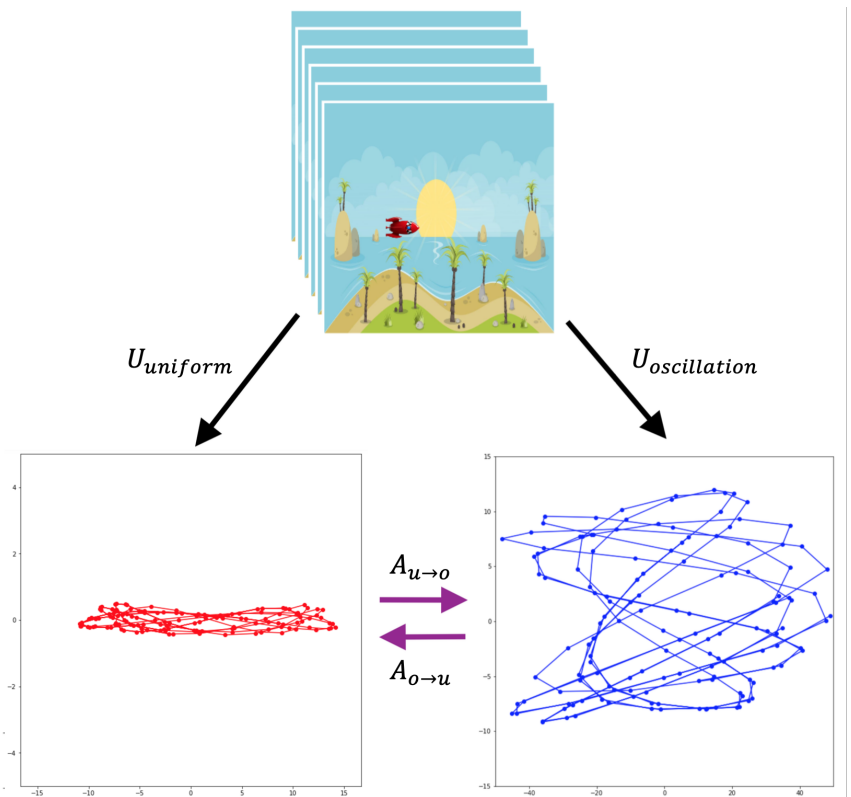

FIG. 6: Video trajectories in the 2D harmonic oscillator potential (top) look different when mapped into the latent space using the encoder trained on that same data (right) than when mapped using the encoder trained on uniform force-free motion (left). However, the two latent spaces are equivalent up to an affine transformation.

implies that the five latent spaces (bottom panels in Figure 4 will generally all be different, since we trained a separate neural network for each case. Specifically, we expect the latent spaces to each differ by some affine transformation $\mathbf{r} \mapsto \mathbf{A r}+\mathbf{a}$ for some constant vector $\mathbf{a}$ and $2 \times 2$ matrix $\mathbf{A}$, since affine transformations do not affect the amount of nonlinearity or acceleration required and thus leave our complexity loss functions $\mathcal{L}^{\text {acc }}$ and $\mathcal{L}^{\text {curv }}$ unchanged.

Figure 6 shows the same sequence of images of the rocket moving in the $2 \mathrm{D}$ harmonic oscillator potential mapped into two latent spaces, learned by training our pregression algorithm on either that same 2D oscillator data (right panel) or on the force-free data (left panel). As expected, they trajectories are seen to differ by an affine transformation. Indeed, the left panel of Figure 7 shows that the latent spaces discovered by all our 5 experiments are interrelated by affine transformations. Here we have mapped all latent space coordinates $\mathbf{r}_{i}, i=1, \ldots, 5$, into a single unified latent space $\mathbf{r}=\mathbf{A}_{i} \mathbf{r}_{i}+\mathbf{a}_{i}$ by introducing five $2 \times 2$ matrices $\mathbf{A}_{i}$ and $2 \mathrm{D}$ translation vectors $\mathbf{a}_{i}$ to match up corresponding rocket positions (each color is associated with an encoder trained on a specific type of motion: force free (red), 2D oscillator (blue), magnetic (yellow), gravitational field (green) and quartic oscillatior (black) and green. Specifically, without loss of generality we take one of the latent spaces (derived from the harmonic oscillator) to the be unified one, so $\mathbf{r}_{1}=\mathbf{r}, \mathbf{A}_{1}=\mathbf{I}, \mathbf{a}_{1}=0$, and solve for the other $\mathbf{A}_{i}$ and $\mathbf{a}_{i}$ by minimizing the total mismatch

$$
M \equiv \sum_{i=2}^{5}\left\langle\ell\left(\left|\mathbf{A}_{i} \mathbf{r}_{i}+\mathbf{a}_{i}-\mathbf{r}_{1}\right|\right)+\ell\left(\left|\mathbf{A}_{i}^{-1}\left(\mathbf{r}_{1}-\mathbf{a}_{i}\right)-\mathbf{r}_{i}\right|\right)\right\rangle,
$$

where the average is over all our rocket images mapped through the the five encoders. If the loss function penalizing mismatch distance were $\ell(r)=r^{2}$, equation 16 would simply be a $\chi^{2}$-minimization determining $\mathbf{A}_{i}$ and $\mathbf{a}_{i}$ via linear regression, except that we have also penalized inaccuracy in the inverse mapping (second term) to avoid biasing $\mathbf{A}_{i}$ low. To increase robustness toward outliers, we instead followed the prescription of [26] by choosing $\ell(r) \equiv$ $\frac{1}{2} \log _{2}\left[1+(r / \epsilon)^{2}\right]$ and minimizing $M$ with gradient descent, using an annealing schedule $\epsilon=10^{1}, 10^{0}, \ldots, 10^{-10}$.
Next, we estimated the velocity $\dot{\mathbf{r}}$ and acceleration $\ddot{\mathbf{r}}$ at each data point by cubic spline fitting to each trajectory $\mathbf{r}(t)$ in the unified latent space, and discovered candidate differential equations of the form $\ddot{\mathbf{r}}=f(\dot{\mathbf{r}}, \mathbf{r})$ using the publicly available AI Feynman symbolic regression package [22, 23]. To eliminate dependence on the cubic spline approximation, we then recomputed the accuracy of each candidate formula $f$ by using it to predict each data point from its two predecessors using the boundary-value ODE solver scipy.integrate.solve_bvp 77], selecting the most accurate formula for each of our five examples.

Applying an affine transformation $\mathbf{r} \mapsto \mathbf{A r}+\mathbf{a}$ to both the data and these equations of course leaves the prediction accuracy unchanged, so we now exploit this to further reduce the total informationtheoretic complexity of our equations, defined as in [26]. Figure 7 (2nd panel) shows a contour plot of the equations complexity as a function of an overall shift (darker means smaller). We observe a clear optimum for the shift vector a, corresponding to eliminating additive constants in the harmonic and quartic oscillator equations. For example, $\ddot{x}=-x$ is simpler than $\ddot{x}=2.236-x$. The 3rd panel of Figure 7 shows the total equation complexity (as a stacked histogram) as a function of an overall rotation of the coordinate axis. We see several minima: the gravitational example likes $45^{\circ}$ rotation because this makes the new horizontal acceleration vanish, but the other examples outvote it in favor of a $0^{\circ}$ rotation to avoid $x y$ cross-terms.

Only three degrees of freedom now remain in our matrix $\mathbf{A}$ : shear (expanding along some axis and shrinking by the inverse factor along the perpedicular axis) and an overall scaling. We apply the vectorSnap algorithm of 23 to discover rational ratios between parameters and then select the shear that maximizes total accuracy. As it can be seen in the contour plot in the right panel of Figure 7 (darker color means smaller values), $\sim 3 \%$ shear is optimal. Finally, we apply the scaling that minimizes total complexity, resulting in these discovered laws of motion for the force-free, gravitational, magnetic, 2D oscillator and quartic oscillator examples, respectively:

$$
\begin{aligned}
\left(\begin{array}{l}
\ddot{x} \\
\ddot{y}
\end{array}\right) & =\left(\begin{array}{l}
0 \\
0
\end{array}\right) \\
\left(\begin{array}{c}
\ddot{x} \\
\ddot{y}
\end{array}\right) & =-\left(\begin{array}{c}
1 \\
1
\end{array}\right) \\
\left(\begin{array}{c}
\ddot{x} \\
\ddot{y}
\end{array}\right) & =\frac{1}{3}\left(\begin{array}{c}
\dot{y} \\
-\dot{x}
\end{array}\right) \\
\left(\begin{array}{c}
\ddot{x} \\
\ddot{y}
\end{array}\right) & =-\frac{1}{9}\left(\begin{array}{c}
4 x \\
y
\end{array}\right) \\
\left(\begin{array}{c}
\ddot{x} \\
\ddot{y}
\end{array}\right) & =-7.3 \times 10^{-6}\left(x^{2}+y^{2}\right)\left(\begin{array}{l}
x \\
y
\end{array}\right)
\end{aligned}
$$

These are in fact exactly the laws of motion used to generate our training set images (up to some noise in the quartic term prefactor), but reexpressed in a five times smaller latent space than the one we used, which further simplifies our formulas (for example, the discovered gravitational acceleration is 1 instead of 5).

\section{SUMMARY}

We have presented a method for unsupervised learning of equations of motion for objects in raw and optionally distorted unlabeled video. This automatic un-distortion may be helpful for modeling real-world video afflicted by stereoscopic projection, lens artifacts, varying lighting conditions, etc., and also for learning degrees of freedom such as 3D coordinates and rotation angles. Our method is in no way limited to video, and can be applied to any timeevolving dataset, say $N$ numbers measured by a set of sensors. Although we focused on dynamics, it could also be interesting to 

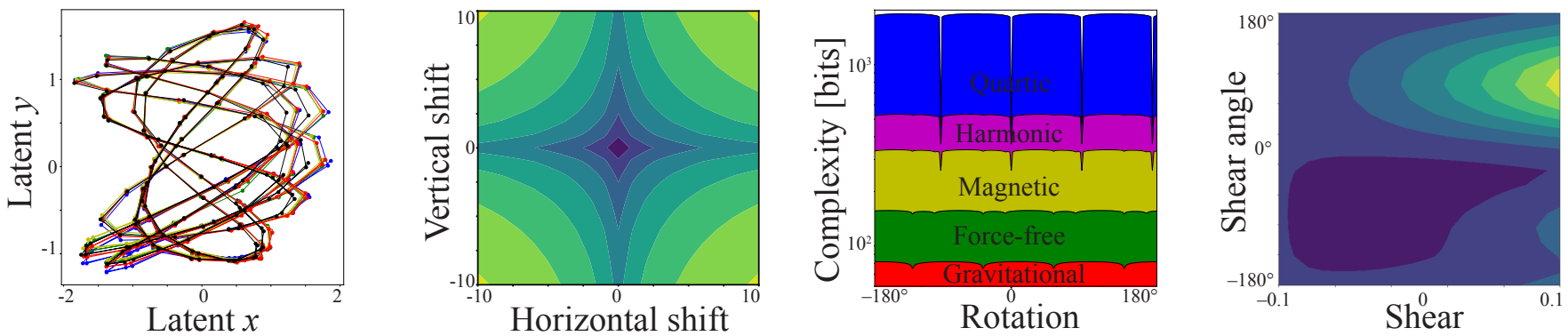

FIG. 7: (Left) The five latent spaces can be unified by using affine transformations. The complexity of the equations in this unified space is minimized when having no further shift (2nd panel) or rotation (3rd panel), but a $\sim 3 \%$ shear (right).

generalize our approach to other situations, by attempting to infer other properties of the system rather than its future state. Another interesting avenue for future work is to explore whether the abovementioned topological intuition provided by knot theory can help improve autoencoders more generally.

The reparametrization invariance of general relativity teaches us that there is an infinite class of coordinate systems that provide are equally valid physical descriptions, and we found a similar reparametrization invariance of our auto-discovered latent space. We broke this degeneracy by quantifying and minimizing the geometric and symbolic complexity of the dynamics. Although different systems were simplest in different coordinate systems, we found that minimizing total complexity for all of them recovered a stan- dard isotropic inertial frame. An interesting topic for future work would be to explore whether our brains' representations of physical systems are similarly optimized to make prediction as simple as possible.

Acknowledgements: The authors with to thank Zhiyu Dong, Jiahai Feng, Bhairav Mehta, Andrew Tan and Tailin Wu for helpful comments, and the Center for Brains, Minds, and Machines (CBMM) for hospitality. This work was supported by Institute for AI and Fundamental Interactions, The Casey and Family Foundation, the Ethics and Governance of AI Fund, the Foundational Questions Institute, the Rothberg Family Fund for Cognitive Science and the Templeton World Charity Foundation, Inc.
[1] Alexandre Koyré. The Astronomical Revolution: Copernicus-Kepler-Borelli. Routledge, 2013.

[2] James P Crutchfield and Bruce S McNamara. Equation of motion from a data series. Complex systems, 1(417452):121, 1987.

[3] Saso Dzeroski and Ljupco Todorovski. Discovering dynamics: from inductive logic programming to machine discovery. Journal of Intelligent Information Systems, 4(1):89-108, 1995.

[4] Elizabeth Bradley, Matthew Easley, and Reinhard Stolle. Reasoning about nonlinear system identification. Artificial Intelligence, 133(1-2):139-188, 2001.

[5] Pat Langley, Dileep George, Stephen D Bay, and Kazumi Saito. Robust induction of process models from timeseries data. In Proceedings of the 20th International Conference on Machine Learning (ICML-03), pages 432-439, 2003.

[6] Michael Schmidt and Hod Lipson. Distilling freeform natural laws from experimental data. science, 324(5923):81-85, 2009.

[7] Randall K McRee. Symbolic regression using nearest neighbor indexing. In Proceedings of the 12th annual conference companion on Genetic and evolutionary computation, pages 1983-1990. ACM, 2010.

[8] Dominic P Searson, David E Leahy, and Mark J Willis. Gptips: an open source genetic programming toolbox for multigene symbolic regression. In Proceedings of the International multiconference of engineers and computer scientists, volume 1, pages 77-80. IMECS Hong Kong, 2010.

[9] Renáta Dubčáková. Eureqa: software review. Ge- netic programming and evolvable machines, 12(2):173178,2011

[10] Sean Stijven, Wouter Minnebo, and Katya Vladislavleva. Separating the wheat from the chaff: on feature selection and feature importance in regression random forests and symbolic regression. In Proceedings of the 13th annual conference companion on Genetic and evolutionary computation, pages 623-630. ACM, 2011.

[11] Michael D Schmidt, Ravishankar R Vallabhajosyula, Jerry W Jenkins, Jonathan E Hood, Abhishek S Soni, John P Wikswo, and Hod Lipson. Automated refinement and inference of analytical models for metabolic networks. Physical biology, 8(5):055011, 2011.

[12] Christopher Hillar and Friedrich Sommer. Comment on the article" distilling free-form natural laws from experimental data". arXiv preprint arXiv:1210.7273, 2012.

[13] Bryan C Daniels and Ilya Nemenman. Automated adaptive inference of phenomenological dynamical models. Nature communications, 6:8133, 2015.

[14] Pat Langley and Adam Arvay. Heuristic induction of rate-based process models. In Twenty-Ninth AAAI Conference on Artificial Intelligence, 2015.

[15] Ignacio Arnaldo, Una-May O'Reilly, and Kalyan Veeramachaneni. Building predictive models via feature synthesis. In Proceedings of the 2015 Annual Conference on Genetic and Evolutionary Computation, pages 983-990, 2015.

[16] Steven L Brunton, Joshua L Proctor, and J Nathan Kutz. Discovering governing equations from data by sparse identification of nonlinear dynamical systems. Proceedings of the National Academy of Sciences, 113(15):3932- 
3937, 2016.

[17] Matthew Guzdial, Boyang Li, and Mark O Riedl. Game engine learning from video. In IJCAI, pages 3707-3713, 2017.

[18] Markus Quade, Markus Abel, J Nathan Kutz, and Steven L Brunton. Sparse identification of nonlinear dynamics for rapid model recovery. Chaos: An Interdisciplinary Journal of Nonlinear Science, 28(6):063116, 2018.

[19] Maciej Koch-Janusz and Zohar Ringel. Mutual information, neural networks and the renormalization group. Nature Physics, 14(6):578, 2018.

[20] Weiwei Kong, Christopher Liaw, Aranyak Mehta, and D Sivakumar. A new dog learns old tricks: Rl finds classic optimization algorithms. ICLR, 2018.

[21] Jiechun Liang and Xi Zhu. Phillips-inspired machine learning for band gap and exciton binding energy prediction. The journal of physical chemistry letters, 10(18):5640-5646, 2019.

[22] Silviu-Marian Udrescu and Max Tegmark. AI Feynman: A physics-inspired method for symbolic regression. Science Advances, 6(16):eaay2631, 2020.

[23] Silviu-Marian Udrescu, Andrew Tan, Jiahai Feng, Orisvaldo Neto, Tailin Wu, and Max Tegmark. Ai feynman 2.0: Pareto-optimal symbolic regression exploiting graph modularity. arXiv preprint arXiv:2006.10782, 2020.

[24] Eugene P Wigner. Invariance in physical theory. Proceedings of the American Philosophical Society, 93(7):521526, 1949.

[25] Yann LeCun, Yoshua Bengio, and Geoffrey Hinton. Deep learning. nature, 521(7553):436-444, 2015.

[26] Tailin Wu and Max Tegmark. Toward an artificial intelligence physicist for unsupervised learning. Physical Review E, 100(3):033311, 2019.

[27] Raban Iten, Tony Metger, Henrik Wilming, Lídia Del Rio, and Renato Renner. Discovering physical concepts with neural networks. arXiv preprint arXiv:180\%.10300, 2018.

[28] MarcAurelio Ranzato, Arthur Szlam, Joan Bruna, Michael Mathieu, Ronan Collobert, and Sumit Chopra. Video (language) modeling: a baseline for generative models of natural videos. arXiv preprint arXiv:1412.6604, 2014.

[29] Vincent Michalski, Roland Memisevic, and Kishore Konda. Modeling deep temporal dependencies with recurrent grammar cells"". In Advances in neural information processing systems, pages 1925-1933, 2014.

[30] Nitish Srivastava, Elman Mansimov, and Ruslan Salakhudinov. Unsupervised learning of video representations using lstms. In International conference on machine learning, pages 843-852, 2015.

[31] Junhyuk Oh, Xiaoxiao Guo, Honglak Lee, Richard L Lewis, and Satinder Singh. Action-conditional video prediction using deep networks in atari games. In Advances in neural information processing systems, pages 28632871, 2015.

[32] Chelsea Finn, Ian Goodfellow, and Sergey Levine. Unsupervised learning for physical interaction through video prediction. In Advances in neural information processing systems, pages 64-72, 2016.

[33] William Lotter, Gabriel Kreiman, and David Cox. Deep predictive coding networks for video prediction and unsupervised learning. arXiv preprint arXiv:1605.08104, 2016.

[34] Francesco Cricri, Xingyang Ni, Mikko Honkala, Emre
Aksu, and Moncef Gabbouj. Video ladder networks. arXiv preprint arXiv:1612.01756, 2016.

[35] Nal Kalchbrenner, Aäron van den Oord, Karen Simonyan, Ivo Danihelka, Oriol Vinyals, Alex Graves, and Koray Kavukcuoglu. Video pixel networks. In Proceedings of the 34th International Conference on Machine Learning-Volume 70, pages 1771-1779. JMLR. org, 2017.

[36] Xiaodan Liang, Lisa Lee, Wei Dai, and Eric P Xing. Dual motion gan for future-flow embedded video prediction. In Proceedings of the IEEE International Conference on Computer Vision, pages 1744-1752, 2017.

[37] Emily L Denton et al. Unsupervised learning of disentangled representations from video. In Advances in neural information processing systems, pages 4414-4423, 2017.

[38] Ruben Villegas, Jimei Yang, Seunghoon Hong, Xunyu Lin, and Honglak Lee. Decomposing motion and content for natural video sequence prediction. arXiv preprint arXiv:1706.08033, 2017.

[39] Vedran Vukotić, Silvia-Laura Pintea, Christian Raymond, Guillaume Gravier, and Jan C Van Gemert. Onestep time-dependent future video frame prediction with a convolutional encoder-decoder neural network. In International Conference on Image Analysis and Processing, pages 140-151. Springer, 2017.

[40] Mohammad Babaeizadeh, Chelsea Finn, Dumitru Erhan, Roy H Campbell, and Sergey Levine. Stochastic variational video prediction. arXiv preprint arXiv:1710.11252, 2017.

[41] Marc Oliu, Javier Selva, and Sergio Escalera. Folded recurrent neural networks for future video prediction. In Proceedings of the European Conference on Computer Vision (ECCV), pages 716-731, 2018.

[42] Zhipeng Liu, Xiujuan Chai, and Xilin Chen. Deep memory and prediction neural network for video prediction. Neurocomputing, 331:235-241, 2019.

[43] Juan Carrasquilla and Roger G Melko. Machine learning phases of matter. Nature Physics, 13(5):431, 2017.

[44] Evert PL Van Nieuwenburg, Ye-Hua Liu, and Sebastian D Huber. Learning phase transitions by confusion. Nature Physics, 13(5):435, 2017.

[45] Evert van Nieuwenburg, Eyal Bairey, and Gil Refael. Learning phase transitions from dynamics. Physical Review B, 98(6):060301, 2018.

[46] Giacomo Torlai and Roger G Melko. Learning thermodynamics with boltzmann machines. Physical Review B, 94(16):165134, 2016.

[47] Tomi Ohtsuki and Tomoki Ohtsuki. Deep learning the quantum phase transitions in random electron systems: Applications to three dimensions. Journal of the Physical Society of Japan, 86(4):044708, 2017.

[48] Vedran Dunjko and Hans J Briegel. Machine learning $\&$ artificial intelligence in the quantum domain: a review of recent progress. Reports on Progress in Physics, 81(7):074001, 2018.

[49] Ilker Yildirim, Kevin A Smith, Mario Belledonne, Jiajun $\mathrm{Wu}$, and Joshua B Tenenbaum. Neurocomputational modeling of human physical scene understanding. In 2nd Conference on Cognitive Computational Neuroscience, 2018.

[50] David Zheng, Vinson Luo, Jiajun Wu, and Joshua B Tenenbaum. Unsupervised learning of latent physical properties using perception-prediction networks. arXiv preprint arXiv:180\%.09244, 2018.

[51] Michael B Chang, Tomer Ullman, Antonio Torralba, and 
Joshua B Tenenbaum. A compositional object-based approach to learning physical dynamics. arXiv preprint arXiv:1612.00341, 2016.

[52] Zhang Zhang, Yi Zhao, Jing Liu, Shuo Wang, Ruyi Tao, Ruyue Xin, and Jiang Zhang. A general deep learning framework for network reconstruction and dynamics learning. Applied Network Science, 4(1):1-17, 2019.

[53] Stuart Russell, Daniel Dewey, and Max Tegmark. Research priorities for robust and beneficial artificial intelligence. Ai Magazine, 36(4):105-114, 2015.

[54] Dario Amodei, Chris Olah, Jacob Steinhardt, Paul Christiano, John Schulman, and Dan Mané. Concrete problems in ai safety. arXiv preprint arXiv:1606.06565, 2016.

[55] Margaret Boden, Joanna Bryson, Darwin Caldwell, Kerstin Dautenhahn, Lilian Edwards, Sarah Kember, Paul Newman, Vivienne Parry, Geoff Pegman, Tom Rodden, et al. Principles of robotics: regulating robots in the real world. Connection Science, 29(2):124-129, 2017.

[56] Peter W Battaglia, Jessica B Hamrick, Victor Bapst, Alvaro Sanchez-Gonzalez, Vinicius Zambaldi, Mateusz Malinowski, Andrea Tacchetti, David Raposo, Adam Santoro, Ryan Faulkner, et al. Relational inductive biases, deep learning, and graph networks. arXiv preprint arXiv:1806.01261, 2018.

[57] Kiran S Bhat, Steven M Seitz, Jovan Popović, and Pradeep K Khosla. Computing the physical parameters of rigid-body motion from video. In European Conference on Computer Vision, pages 551-565. Springer, 2002.

[58] Jonathan Tompson, Kristofer Schlachter, Pablo Sprechmann, and Ken Perlin. Accelerating eulerian fluid simulation with convolutional networks. In Proceedings of the 34th International Conference on Machine LearningVolume 70, pages 3424-3433. JMLR. org, 2017.

[59] Peter Y Lu, Samuel Kim, and Marin Soljačić. Extracting interpretable physical parameters from spatiotemporal systems using unsupervised learning. arXiv preprint arXiv:190\%.06011, 2019

[60] Jiajun Wu, Joseph J Lim, Hongyi Zhang, Joshua B Tenenbaum, and William T Freeman. Physics 101: Learning physical object properties from unlabeled videos. In $B M V C$, volume 2, page 7, 2016.

[61] Adam Santoro, David Raposo, David G Barrett, Mateusz Malinowski, Razvan Pascanu, Peter Battaglia, and Timothy Lillicrap. A simple neural network module for relational reasoning. In Advances in neural information processing systems, pages 4967-4976, 2017.

[62] Rosanne Liu, Joel Lehman, Piero Molino, Felipe Petroski Such, Eric Frank, Alex Sergeev, and Jason Yosinski. An intriguing failing of convolutional neural networks and the coordconv solution. In Advances in Neural Information Processing Systems, pages 9605-9616, 2018.

[63] Jessica B Hamrick, Kelsey R Allen, Victor Bapst, Tina Zhu, Kevin R McKee, Joshua B Tenenbaum, and Peter W Battaglia. Relational inductive bias for physical construction in humans and machines. arXiv preprint arXiv:1806.01203, 2018.

[64] Nicholas Watters, Daniel Zoran, Theophane Weber, Peter Battaglia, Razvan Pascanu, and Andrea Tacchetti. Visual interaction networks: Learning a physics simula- tor from video. In Advances in neural information processing systems, pages 4539-4547, 2017.

[65] Aaron van den Oord, Yazhe Li, and Oriol Vinyals. Representation learning with contrastive predictive coding. arXiv preprint arXiv:1807.03748, 2018.

[66] Samuel Greydanus, Misko Dzamba, and Jason Yosinski. Hamiltonian neural networks. In Advances in Neural Information Processing Systems, pages 15353-15363, 2019.

[67] Hervé Bourlard and Yves Kamp. Auto-association by multilayer perceptrons and singular value decomposition. Biological cybernetics, 59(4-5):291-294, 1988.

[68] Yann LeCun, Bernhard Boser, John S Denker, Donnie Henderson, Richard E Howard, Wayne Hubbard, and Lawrence D Jackel. Backpropagation applied to handwritten zip code recognition. Neural computation, 1(4):541-551, 1989.

[69] Geoffrey E Hinton and Richard S Zemel. Autoencoders, minimum description length and helmholtz free energy. In Advances in neural information processing systems, pages 3-10, 1994.

[70] Geoffrey E Hinton and Ruslan R Salakhutdinov. Reducing the dimensionality of data with neural networks. science, 313(5786):504-507, 2006.

[71] Yoshua Bengio, Aaron C Courville, and Pascal Vincent. Unsupervised feature learning and deep learning: A review and new perspectives. CoRR, abs/1206.5538, 1:2012, 2012.

[72] Diederik P Kingma and Max Welling. Auto-encoding variational bayes. arXiv preprint arXiv:1312.6114, 2013.

[73] Yoshua Bengio. Deep learning of representations: Looking forward. In International Conference on Statistical Language and Speech Processing, pages 1-37. Springer, 2013.

[74] Irina Higgins, Loic Matthey, Arka Pal, Christopher Burgess, Xavier Glorot, Matthew Botvinick, Shakir Mohamed, and Alexander Lerchner. beta-vae: Learning basic visual concepts with a constrained variational framework. ICLR, 2(5):6, 2017.

[75] Alessandro Achille and Stefano Soatto. Information dropout: Learning optimal representations through noisy computation. IEEE transactions on pattern analysis and machine intelligence, 40(12):2897-2905, 2018.

[76] EC Zeeman. Unknotting spheres. Annals of Mathematics, pages 350-361, 1960.

[77] Pauli Virtanen, Ralf Gommers, Travis E. Oliphant, Matt Haberland, Tyler Reddy, David Cournapeau, Evgeni Burovski, Pearu Peterson, Warren Weckesser, Jonathan Bright, Stéfan J. van der Walt, Matthew Brett, Joshua Wilson, K. Jarrod Millman, Nikolay Mayorov, Andrew R. J. Nelson, Eric Jones, Robert Kern, Eric Larson, CJ Carey, İhan Polat, Yu Feng, Eric W. Moore, Jake Vand erPlas, Denis Laxalde, Josef Perktold, Robert Cimrman, Ian Henriksen, E. A. Quintero, Charles R Harris, Anne M. Archibald, Antônio H. Ribeiro, Fabian Pedregosa, Paul van Mulbregt, and SciPy 1. 0 Contributors. SciPy 1.0: Fundamental Algorithms for Scientific Computing in Python. Nature Methods, 17:261-272, 2020. 